BULL. AUSTRAL. MATH. SOC.

VOL. 32 (1985), 375-378.

\title{
A STRENGTHENED TOPOLOGICAL CARDINAL INEQUALITY
}

\section{Sun SHU-HaO and :IANG Yan-Ming}

A new cardinal inequality, $|K(X)| \leq 2^{L^{\star}(X) \cdot p s w(X)}$, is proved in this paper. It strengthens the result of D.K. Burke and R. Hodel that $|K(X)| \leq 2^{e(X) \cdot p s w(X)}$.

A bound on the number of compact sets in a topological space is given by D.K. Burke and R. Hodel [1]: for every $T_{1}$-space $X$, we have

$$
|K(X)| \leq 2^{e(X) \cdot p s w(X)} .
$$

Here, $|K(X)|=\mid\{C: C$ is a compact subset of $X\} \mid$;

$e(X)=\sup \{|D|: D$ is a closed discrete subspace of $X\}+\omega ;$ and psw $(X)=\min \{k$ : there exists some separating open cover $U$ of $X$ with ord $(x, U) \leq k$ for all $x \in X$ \}. (The cover $U$ of $X$ is separating if $\cap\{U U: x U\}=\{x\}$ for all $x \in X$, and $\operatorname{ord}(x, U)=|\{U \in U: x \in U\}|$.) For this and related results, see the survey paper Hodel [4]. We generalize this result in this paper.

First, we give a definition as follows:

DEFINITION. For every topological space $X$, the * Lindelof number of $\underline{X}$, denoted by $L^{\star}(X)$, is defined by:

$L^{*}(X)=\min \{k$ : for every open cover $U$. of $X$, there exists

$A \subseteq X$ with $|A| \leq k$ such that $\cup \operatorname{st}(x, U)=X\}$.

Received 26 March, 1985.

Copyright Clearance Centre, Inc. Serial-fee code: 0004-9727/85 $\$ A 2.00+0.00$. 


\section{LEMMA 1. (Burke's Lemma, [3, Theorem 1.1])}

If $\left\{A_{\alpha}: \alpha \in \Lambda\right\}$ is an indexed colzection of sets in which every member has cardinality $\leq \lambda$, where $|\Lambda|>2^{\lambda}$, and each $A_{\alpha}$ is a disjoint union of two subsets $A_{\alpha}^{\prime}, A_{\alpha}^{\prime \prime}$, then there is a set $\Lambda^{\prime} \subseteq \Lambda$ such that $\left|\Lambda^{\prime}\right|>2^{\lambda}$ and $A_{\alpha}^{\prime} \cap A_{\beta}^{\prime \prime}=\varnothing$ when $\alpha, \beta \in \Lambda^{\prime}$.

The main theorem in this paper is as follows:

THEOREM. For every $T_{1}$-space $X$, we have

$$
|K(X)| \leq 2^{L *(X) \cdot p s w(X)} .
$$

Proof. The first step is to show that for $x \in T_{1}$ we have $|X| \leq 2^{L^{\star}(X) \cdot p s w(X)}$, using Burke's Lemma.

Let $L^{*}(X) \cdot p \operatorname{sw}(X)=\lambda$, then $p \operatorname{sw}(X) \leq \lambda$ and $L^{*}(X) \leq \lambda$. Thus there is an open cover $U$ of $X$ such that $n\{U \in U: x \in U\}=\{x\}$ and ord $(x, U) \leq \lambda$ for all $x \in X$. We first construct the sets $A_{y}=A_{y}^{\prime} \cup A_{y}^{\prime \prime}$ such that $A_{y}^{\prime} \cap A_{y}^{\prime \prime}=\varnothing$ and $\left|A_{y}\right| \leq \lambda$ for all $y \in X$ as subsets of $X$. In fact, $\{U \in U: y \in U\}$ can be indexed and denoted by $\left\{U_{\alpha}\right\}_{\alpha<\lambda}$. Let $V=\{U \epsilon U \mid y \Phi U\}$ and $U_{\alpha}=V u\left\{U_{\alpha}\right\}$ for $\alpha<\lambda$. Since $L^{\star}(X) \leq \lambda$, there exists some $B_{\alpha} \subset X$ such that $\left|B_{\alpha}\right| \leq \lambda$ and $\underset{x \in B_{\alpha}}{U} \operatorname{st}\left(x, U_{\alpha}\right)=X$. Since $\operatorname{st}\left(x, u_{\alpha}\right) \subset \operatorname{st}(x, v) \cup U_{\alpha}$ when $x \neq y$, but $\operatorname{st}\left(y, u_{\alpha}\right)=U_{\alpha}$, then we have $\underset{x \in B_{\alpha}}{\cup} s t(x, V) \cup U_{\alpha}=X$, and therefore $\underset{x \in B_{\alpha}}{\cup} s t(x, V)=X-U_{\alpha}$. Let $B(y)=\underset{\alpha<\lambda}{\cup} B_{\alpha}$, then $|B(y)| \leq \lambda \cdot \lambda=\lambda$. Then $\underset{x \in B(y)}{U} \operatorname{st}(x, V)=$ $\underset{\alpha<\lambda}{U} \underset{x \in B_{\alpha}}{U} \operatorname{st}(x, V)>\underset{\alpha<\lambda}{U}\left(X-U_{\alpha}\right)=X-\underset{\alpha<\lambda}{\cap} U_{\alpha}=X-\{y\}$. Since $y \notin \underset{x \in B(y)}{U}$ st $(x, V)$, we have $\underset{x \in B(y)}{U} \operatorname{st}(x, V)=X-\{y\}$. since $|\underset{x \in B(y)}{U}\{U \in V \mid x \in U\}| \leq \lambda \cdot \lambda=\lambda$ and $\operatorname{ord}(y, U) \leq \lambda$, we can define the set $A_{y}=A_{y}^{\prime} \cup A_{y}^{\prime \prime}$, where $A_{y}^{\prime}=\underset{x \in B_{y}}{U}\{U \in U: x \in U\}$ and $A_{y}^{\prime \prime}=\{U \in U: y \in U\}$. It is clear that $A_{y}^{\prime} \cap A_{y}^{\prime \prime}=\emptyset$ and $\left|A_{y}\right| \leq\left|A^{\prime}\right|+\left|A^{\prime \prime}\right| \leq \lambda+\lambda=\lambda$. 
Now, we have defined the sets $A_{y}$ for all $y \in X$. We can obtain $|X| \leq 2^{\lambda}$, immediately. Otherwise, $|X|>2^{\lambda}$. Let $A=\left\{A_{x}\right\}_{x \in X}$. Then, by Burke's Lemma, there is a subset $X^{\prime} \subset X$ such that $\left|X^{\prime}\right|>2^{\lambda}$ and $A_{x}^{\prime} \cap A_{y}^{\prime \prime}=\varnothing$ for any $x, y \in X^{\prime}$. But this is impossible, because $y \in X-\{x\}=\underset{x^{\prime} \in B(x)}{U} \operatorname{st}\left(x^{\prime}, U\right)$ where $V^{\prime}=\{U \in U \mid X \notin U\}$, whenever $x \neq y$. However, there exists some $U \in A_{x}^{\prime}$ such that $y \in U$ and, of course, $U \in A_{y}^{\prime \prime}$ so $A_{x}^{\prime} \cap A_{y}^{\prime \prime}=\varnothing$, a contradiction. This contradiction shows that we must have $|X| \leq 2^{\lambda}=2^{L^{*}(X) \cdot p s w(X)}$.

A standard argument now establishes that $|K(X)| \leq 2^{L^{\star}(X) \cdot p \operatorname{sw}(X)}=2^{\lambda}$, for example see Hodel [4, proof of Theorem 9.3].

REMARK. The definition of $L^{\star}(X)$ was first introduced by Dai MuMing [5], and an independent proof of the result $|X| \leq 2^{L^{\star}}(X) \cdot p s w(X)$ is given in [5]. But our argument is simpler than the original argument.

COROLLARY 1. (D.K. Burke and R. Hode1 [1, Theorem 4.4]) For every $T_{1}$-space $X$, we have

$$
\mid K(X) 1 \leq 2^{e(X) \cdot p \operatorname{sw}(X)}
$$

Proof. It is sufficient to show $e(X) \geq L^{*}(X)$. In fact, for every open cover $U$ of $X$, consider the maximal subset $A \subseteq X$ satisfying the following property (*).

$(*)$ : for all $x, y \in A$ if $x \neq y$ then $x$ \& st $(y, U)$. clearly, $X=\underset{y \in A}{U} \operatorname{st}(y, U)$. Otherwise, there exists an $x_{0} \in X-\underset{y \in A}{U} \operatorname{st}(y, U)$. But then $s t\left(x_{0}, U\right) \cap A=\varnothing$ and $A \cup\left\{x_{0}\right\}$ satisfies the property (*), contradicting the fact that $A$ is maximal. By definition, $L^{\star}(X) \leq|A|$. To show that $A$ is a closed discrete subspace of $X$, note $A$ is discrete since st $(a, U)$ is open and $\{a\}=\operatorname{st}(a, u) \cap A$ for all $a \in A$ and $A$ is closed since for all $x \in X-A$, there exists an $a_{0} \in A$ such that $x \in \operatorname{st}\left(a_{0}, u\right)$ and $X$ is a $T_{1}$-space, so $s t\left(a_{0}, U\right)-\left\{a_{0}\right\}$ is open and it is disjoint from $A$. By definition, we have $e(X) \geq|A| \geq L^{\star}(X)$. 
EXAMPLE 1. The Niemytzki plane $X$ is separable, so $L^{\star}(X)=\omega_{0}$, but it contains a closed discrete subspace of cardinality $c$, and therefore $e(X) \geq c>\omega_{0}=L^{*}(X)$.

EXAMPLE 2. Let $Y=N^{c}$, where $N$ is the discrete countable space. By the Hewitt-Marczewski-Pondiczery theorem, $d(Y)=\omega_{0}$, and hence

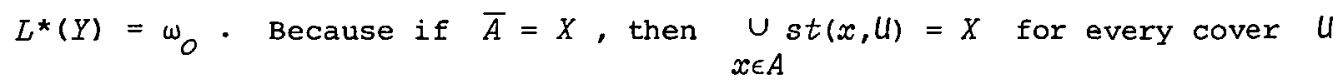
of $X$. Engelking [2] has proved that the space contains a closed discrete subspace cardinality of $c$, and so $e(Y) \geq c$. Thus $e(Y)>L^{\star}(Y)$, also.

These examples show that the theorem in this paper is a significant extension for Burke and Hodel's result [1].

COROLLARY 2. For every $T_{1}$-space $X$, we have

$$
|K(X)| \leq 2^{d(X) \cdot p \sin (X)}
$$

\section{References}

[1] D.K. Burke and R. Hodel, "The number of compact subsets of a topological space", Proc. Amer. Math Soc. 8 (1976), 363-368.

[2] R. Engelking, "General Topology", (Warsawa 1977), 181.

[3] D.K. Burke, A note on R.H. Bing's example $G$, in Topology Conference Virginia Polytechnic Institute and State University, March (1973) (Lecture Notes in Mathematics 375 springer-Verlag 1974), 47-52.

[4] F.R. Hodel, "Cardinal Functions I", Handbook of Set-Theoretic Topology, ed. K. Kunen and J.E. Vaughan (North-Holland, 1984), $1-61$.

[5] Dai MuMing, "A topological space cardinal inequality involving the *Lindelöf number", Acta Mathematica Sinica, 26 (1983), 731-735.

Department of Basic Teaching,

Shanghai Institute of Mechanical

Engineering,

Shanghai, PRC.
Department of Mathematics, Shanghai Normal University, Shanghai, PRC. 